\title{
Integration of virtual and real document organization
}

\author{
Thomas Seifried $^{1}$ \\ ${ }^{1}$ Upper Austria University \\ of Applied Sciences \\ Hagenberg, Austria \\ $\{$ thomas.seifried, \\ michael.haller\}@fh- \\ hagenberg.at
}

Matthew Jervis ${ }^{2}$

Nicolas Villar ${ }^{3}$

${ }^{2}$ The University of Waikato

Hamilton, New Zealand

$\{\mathrm{mgj} 3$,

masood\}@cs.waikato.ac.nz

\author{
Michael Haller ${ }^{1}$
}

Masood Masoodian ${ }^{2}$

${ }^{3}$ Lancaster University

Lancaster, UK

villar@comp.lancs.ac.uk

\begin{abstract}
In most work environments people archive both the real and digital versions of their documents. But unlike the digital world, in the physical world locating a document can become a very time consuming task. The reason for this is the lack of a direct connection between the physical and digital versions of documents.
\end{abstract}

The Smart Filing System combines the benefits of the digital and the real world providing an augmented filing cabinet. The system benefits by linking the physical world with the digital desktop world. In our setup, we implemented an addin for MS OneNote ${ }^{\mathrm{TM}}$. Furthermore, real folders and cabinets are extended with devices for direct input and output. This allows us to search and browse through digital documents using MS OneNote ${ }^{\mathrm{TM}}$. Simultaneously, our system also allows a feedback in the physical world, by highlighting the corresponding folder in the filing cabinet. In this paper we describe the hardware and software implementation our prototype system, and present the results of a preliminary pilot study of its use.

\section{Author Keywords}

smart filing system, tangible folder, interactive folder, digital pen, physical interfaces, tangible interfaces

\section{ACM Classification Keywords}

H5.2 [Information interfaces and presentation]: User Interfaces. Physical user interfaces.

\section{INTRODUCTION}

Despite the many past predictions made about the paperless office of the 21 st century, paper documents, in their various forms, remain an integral part of today's office work environments [12]. In fact in recent times the number of printed paper documents has greatly increased, rather than decreasing or disappearing altogether. Despite this increase in the volume of printed documents, the mechanisms for storage and retrieval of paper documents have remained virtually unchanged over the past 100 years, and still rely heavily on the use of physical folders and filing cabinets. Various forms of filing cabinets, such as paper, document, and pigeonhole cabinets have been in wide-spread use since the mid-nineteenth century; the more well-known form of filing cabinet, often referred to as the vertical filing cabinet, was invented by Seibels in 1898 [11](page 66). Although some cosmetic changes have been made in terms of the material used to make filing cabinets (e.g. wood, metal, steel, etc.), varying their size, and so on, their functionality remains the same.

Furthermore, because of the limited capabilities of the original filing cabinet system, it has been necessary to develop, sometimes rather complicated, filing systems to facilitate the organization, storage and retrieval of documents from filing cabinets. Additionally, the utility of most such systems is often closely linked to the memory of the person primarily responsible for organizing and using the system, which can be problematic when that person is not available or leaves the organization.

With the development of personal computers and their widespread use over the past two decades, a parallel world of document management has emerged in the form of "desktop" windowing systems with virtual folders, files, and documents. However, although the virtual desktop has inherited the idea of documents and folders from the physical office, in a computer environment advanced storage, search and retrieval functionalities have been developed to allow better management of electronic documents.

In a modern office environment the physical and digital document management systems (i.e. the filing system and computer) remain largely disconnected. Even when digital and physical copies of the same or related documents exist in both systems there is often no obvious manageable connection between them, and none of the advanced digital document management tools and techniques can be applied to their physical counterparts. For instance, unlike the digital world, where finding a document requires using a simple 
searching tool on the computer, in the physical world locating a document can become a time consuming task. This is particularly true if the searching criteria for a physical document are not easy to specify (e.g. looking for a document containing a particular keyword). While documents found on a computer using a search tool can be accessed directly by just clicking on a button, physical file organization systems do not provide for a comparatively easy-search mechanism. In addition on the computer, videos, audio tracks and other multimedia content can be stored, searched, and displayed in exactly the same manner without difficulty, but this is not true for physical documents, books, videotapes, CDs and so on.

Using existing technology, at present the only way of managing some association between the physical and digital documents is by creating and duplicating the exact storage structures in both worlds, for instance by naming the folders and documents the same, using the same hierarchy of folders, etc. This often requires printing multiple copies of electronic documents, storing them in different physical folders and so on, which is clearly not very efficient and far less than satisfactory.

In this paper, we present the Smart Filing System, a tangible interactive filing cabinet system which combines the properties of physical folders with a virtual (digital) Document Management System (DMS), and narrows the gap between the physical and digital worlds of organization and usage of documents. Our system applies a technique similar to that used in some car parks to show available parking spaces by turning on lights associated with free spaces (cf. figure 1) as a way of finding folders in an archive [4].

\section{RELATED WORK}

The vast majority of research in document management systems has either focused on developing electronic tools which attempt to create an almost mythical paperless office environment, or aim to provide for better means of tagging and/or annotating physical paper documents. There are however, a number of more recent systems which are designed to reduce the gap between physical and electronic documents.

One such system is the Digital Desk [14] in which the paper documents are augmented by projected electronic content. This idea has further been developed into EnhancedDesk [9] which allows displaying related multimedia content next to a textbook. Another similar system is PapierCraft which uses digital pens to allow manipulation of digital documents on real printouts [10]. All these systems, however, offer useful solutions in dealing with single documents only.

Systems that facilitate dealing with many documents, and can therefore be called true document management systems, are even fewer than those dealing with augmenting single documents. PaperWorks [8], developed by Xerox, is one such system. PaperWorks has been designed to integrate real paper into an electronic paper workflow, where the paper acts as an input device by filling out pre-defined forms and digitalizing them by using fax or scanners. This makes

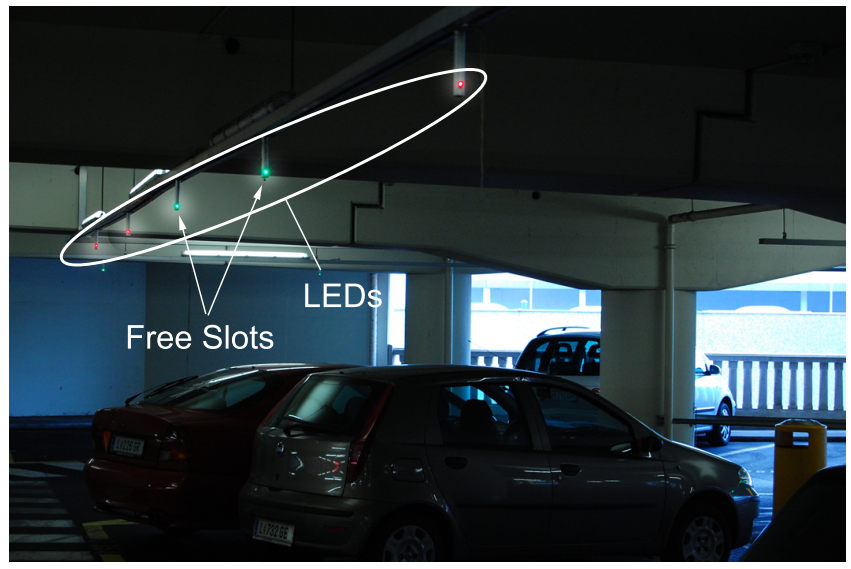

(a)

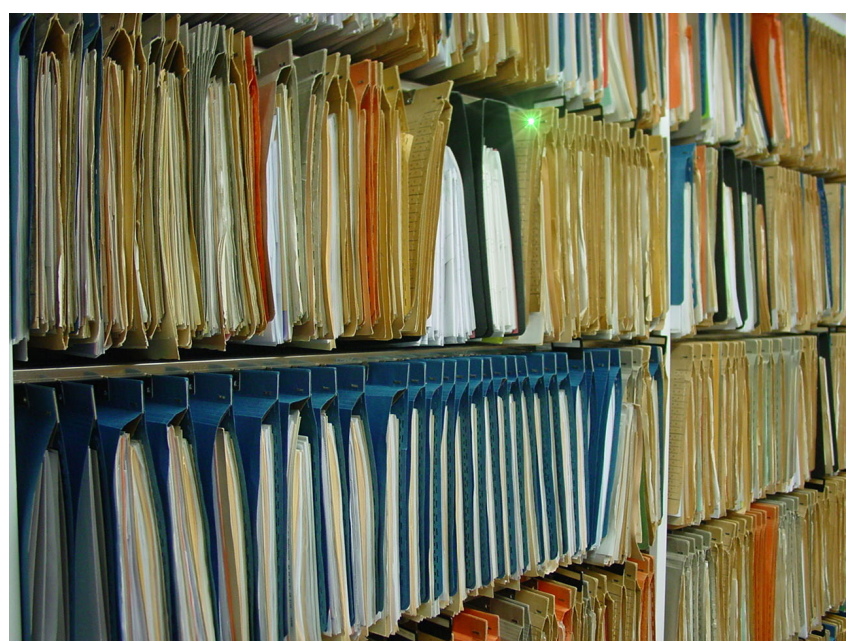

(b)

Figure 1. Using the car park free space indicator technique for finding folders in a filing cabinet.

the task of importing real documents into the electronic document management system easier, but does not provide a link between the physical and electronic versions of the documents.

Another Xerox system [2] utilizes RFID tags on paper documents to connect them to their virtual counterparts. Based on this, AbuSafiya and Mazumadar [1] proposed a model to integrate real paper into the document workflow of a virtual DMS. Using RFID, a link between virtual and real documents is established. RFID readers facilitate the identification and localization of real documents within the reach of the antennas.

Ishii et al. [7] proposes Tangible Interfaces as a convenient way for people to interact with computer systems. Similar to this work, the project Phidgets[5] facilitates real devices (e.g. small buttons, sliders, knobs) to control applications. In this work such physical controls are linked to existing application widgets. However, those physical controls are not mounted on daily-life objects (e.g. real folders) but create a completely new user interface. 


\section{SMART FILING SYSTEM}

The Smart Filing System is rather unique in that it connects physical and digital documents by electronically enhancing filing cabinets and folders, and providing softwarelevel mechanisms for management and retrieval of both digital and paper documents through a single unified document management system. Unlike other works, this system focuses on linking the organization of physical and digital documents, rather than linking the documents themselves. The Smart Filing System consists of two connected parts: the electronically augmented physical filing cabinets and folders, and the digital software client for structuring, controlling, and searching the physical as well as digital documents. Figure 6 shows these two components of the Smart Filing System.

Our system is designed to support a typical document workflow (depicted in figure 2) in which physical documents are just as important as virtual computer documents. The main reason for this is that although we are living in a digital world, people still prefer to print out documents [12]; because paper still has a lot of advantages: it is easy to navigate, gives a fast overview, is easy to annotate, is socially well accepted, and it doesn't need any power. Moreover, in some cases a real printout is necessary and has to be archived (e.g. in medical reports, certifications etc.).

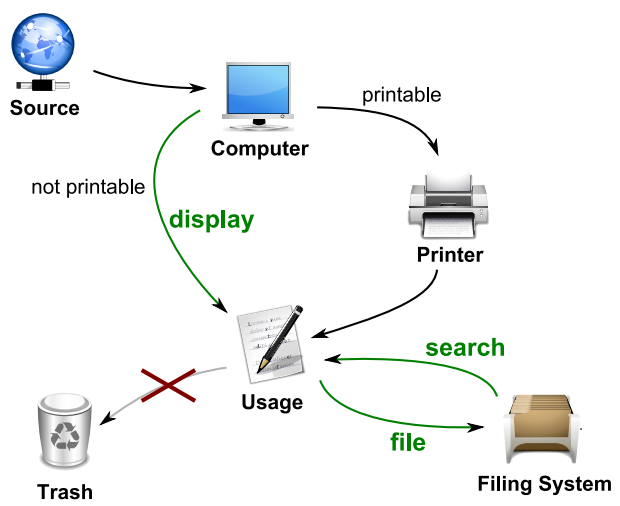

Figure 2. A typical document workflow.

On the other hand, we often also have to work with information and documents which cannot be printed (e.g. movie clips, audio files, $3 \mathrm{~d}$ content). Moreover, searching for documents in large physical archives can be cumbersome. Therefore, our approach is to combine the advantages of the physical folders with the advantages of the digital world.

Figure 3 shows the basic configuration of a Smart Filing System, which consists of two major parts:

- a computer with the document management system, and

- one or more filing cabinets equipped with digital features.

Our prototype implementation of the Smart Filing System also includes an optional interactive table, which allows an

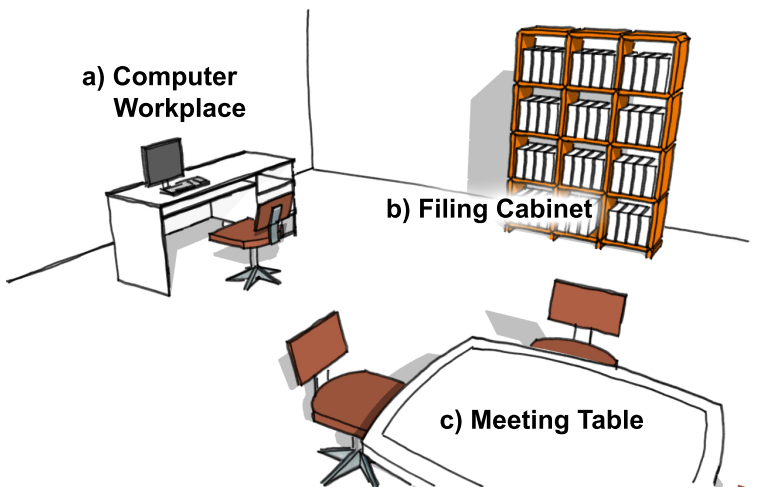

Figure 3. The core of the hardware setup is the digital filing cabinet with its interactive folders and with the optional interactive meeting table.

intuitive interaction and handling of virtual documents.

Each enhanced filing cabinet contains a collection of interactive folders (as shown in figure 6) which are used to store real printouts. A typical filing cabinet contains multiple interactive folders (see figure 4), each containing real printouts. The folders themselves are stored in a certain way, e.g. based on their names.

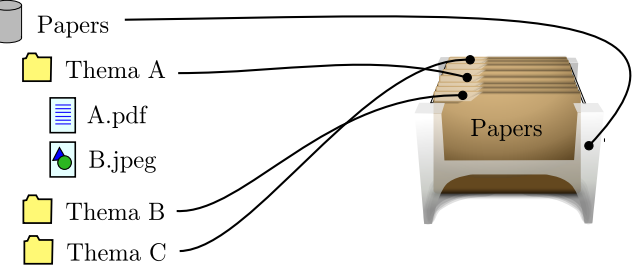

Figure 4. Smart Filing System provides a link between virtual and real organization-level elements (folders and filing cabinets).

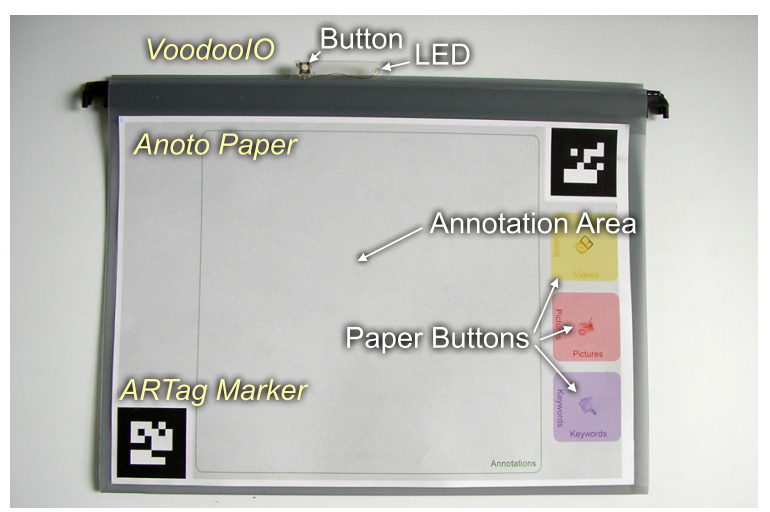

Figure 5. Prototype of an interactive folder.

Each folder has a simple user interface in the form of an LED and a push-button, which are mounted on the tab at the top of the folder (figure 5). The filing cabinet provides a communication medium for the folders (figure 7). When folders are placed in the cabinet their hooks sit on the horizontal metals rails, making an electrical connection. This 


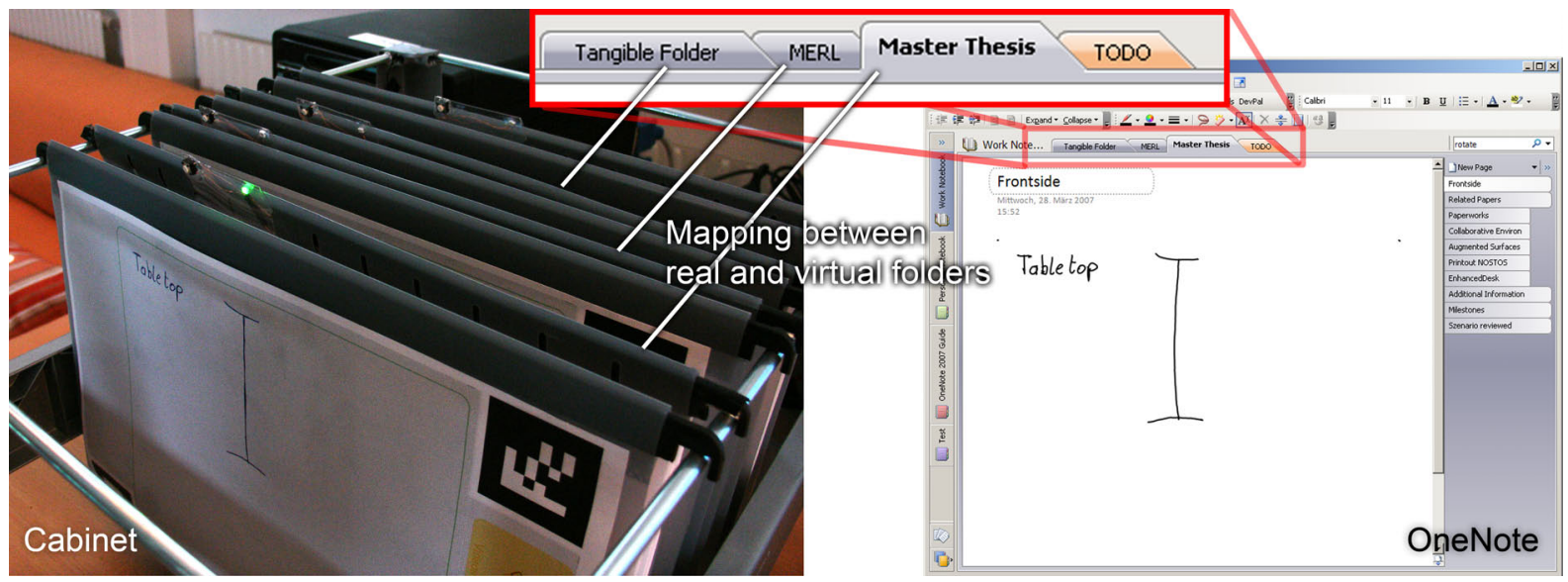

Figure 6. The electronic version of documents is often organized the same way as physical ones. Hence, there is the same folder structure on the computer containing the same documents as on the physical counterpart.

forms a simple network bus allowing the cabinet to communicate with the folders. Each folder has a unique address, allowing individual folders to be identified within the cabinet. A USB controller is used to connect the cabinet to a computer. The cabinet itself incorporates several electronic devices, including a button and a rotary dial mounted on the front side. When folders are added/removed, or the devices on them manipulated, events are passed on to the application software. The application software can also control the state of the folder mounted LEDs.

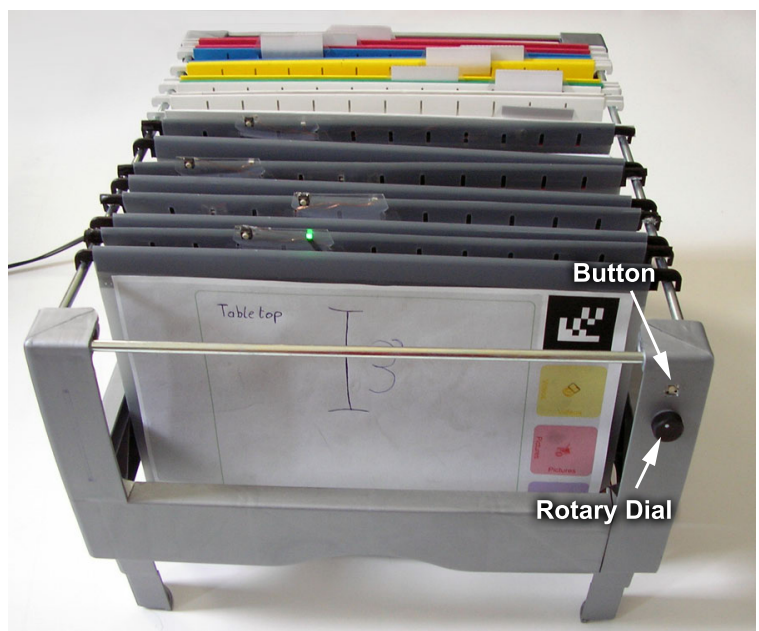

Figure 7. The digital filing cabinet.

On the front side of each folder (figure 5) we also put an A4sized Anoto paper ${ }^{1}$, which provides for a fast and easy interaction using digital pens. Although the Anoto technology has been available for more than five years it is only recently that it has became possible to use a Bluetooth connection to retrieve the pen data in real-time.

In our setup, users can make direct notes on top of the folder (e.g. quick annotations) which are sent wirelessly to the

\footnotetext{
${ }^{1}$ http://www.anoto.com
}

desktop application over Bluetooth. These notes are usually used for describing the content of a physical folder. By using the digital pen such notes are available on the virtual folder too. In addition to the Anoto pattern, two unique ARTag markers [3] attached to the front side of each folder allow, it to be tracked once placed on the optional interactive tabletop $^{2}$.

\section{Smart Filing Technology}

The filing cabinet and folders that make up the Smart Filing System are based on VoodooIO technology. VoodooIO is a malleable control structure, an input device that comprises "a set of controls that can be freely arranged on control areas" [13]. Similarities in the structure of VoodooIO and the Smart Filing System mean that VoodooIO technology makes a good base for development (see figure 8).

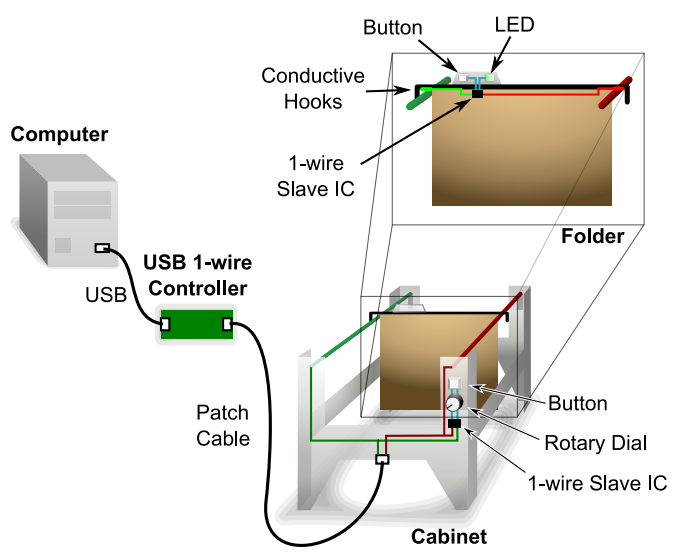

Figure 8. Wiring of the Smart Filing System.

The VoodooIO control area provides the connection medium for the controls, and is known as the network substrate. Controls are attached to the substrate by means of co-axial pins

\footnotetext{
${ }^{2}$ The interactive tabletop system is not described here as this paper's focus is on the filing system
} 
that contact two separate conductive layers in the substrate. VoodooIO uses 1-wire technology developed by Dallas-Ma$\mathrm{xim}^{3}$ to provide communication over this two point connection. 1-wire technology allows devices to be powered and communicate using a single wire; a ground wire is also needed, meaning two connections are actually required. Each control contains at least one 1-wire slave IC, which provides each VoodooIO control with a unique ID, and an interface between the 1-wire network and the physical controls (such as buttons, dials and sliders). The substrate is connected to a computer via a USB 1-wire controller, which acts as master to the 1-wire network.

The software component of VoodooIO, known as the VoodooIO service, is responsible for controlling the 1-wire network via the USB controller, while providing a high-level abstracted interface to application software. The service polls the 1-wire network for the addition, removal and manipulation of slave devices and communicates these as events to higher layers. It provides a TCP service to which applications can connect to receive notification of addition, removal and manipulation of controls, as well as control output devices, such as LEDs on the controls.

While the smart filing system is based on VoodooIO technology, it applies this technology in a novel way. In place of the network substrate, the digitally enhanced filing cabinet is used as the network medium; and folders, rather than controls, are the network nodes. The logical structure of the nodes remains mostly unchanged. A modified version of the VoodooIO service is used at the software level.

The smart folders are augmented with 1-wire slave ICs to give them a unique ID and provide an interface to their button and LED. The filing cabinet is enhanced to provide the means of connecting folders to the 1-wire network. The filing cabinet has horizontal metal rails running its length; when a folder is placed in the filing cabinet, hooks on each side sit on these rails. This combination of hooks and rails provides two points of electrical connection, analogous to the two conductive layers of substrate in VoodooIO. One of the rails is wired to the power/data line of the 1-wire network, while the other is wired to ground. Using the cabinet as the 1-wire network medium makes it possible to discover when folders are added to/removed from the cabinet.

The hooks of the folders are part of the crossmembers that run laterally along the top of each folder. These crossmembers are plastic, rather than metal, to prevent them from shorting the rails together. Instead, the hooks of the rear crossmember are wrapped in conductive material, and this is connected to the 1-wire IC.

The button and LED on each folder provide interactivity to the user. Each folder has a single 1-wire slave IC which has two programmable input/output (PIO) pins, and the button and LED connect these. When the button is pressed this generates an event which is transmitted to the application software. The application software can also set the state of

\footnotetext{
${ }^{3}$ www.maxim-ic.com/1-Wire
}

the LED. As events from these devices are dealt with at the application software layer, their actual role is irrelevant at this low layer.

The cabinet itself is a node on the 1-wire network. It connects internally to the same bus as the folders. Like the folders, each cabinet has a unique ID. Additionally, the cabinet provides a button and dial as input devices. As with the I/O devices on the folders, the function of the button and dial is defined at the application software layer.

The Smart Filing System utilizes the VoodooIO service to provide the link between the hardware and the application software. The service has been optimized to quickly detect addition/removal of folders, and take into account the intermittent connectivity caused by the hook/rail connection mechanism.

\section{OneNote Client Application}

Our hardware setup is linked together using Microsoft OneNote 2007 running on a computer ${ }^{4}$. OneNote supports note taking, information gathering, and easy multi-user collaboration. Therefore, it is well-suited for the management, searching, and retrieval of virtual documents in our setup. VoodooIO and Anoto SDKs facilitate the communication with the electronically augmented folders and the enhanced filing cabinet.

For structuring, controlling, and searching the physical as well as digital documents a software client has been developed. Those tasks are performed by a C\#-based OneNote Add-in. It provides an enhanced search feature and interaction with VoodooIO devices mounted on the cabinet and the folders and the digital pens.

The enhanced search feature extends the usual OneNote search functionality to display search results not only on the computer screen but also on the real folders. Furthermore the augmentations on the folder are utilized for three different functions:

- The button enables navigation between virtual folders in OneNote.

- The LED is used to display search results or the actual displayed folder in OneNote.

- Anoto paper and pen are used for adding annotations to the front of the physical as well as the virtual folder.

First of all, these functions require the folders to be distinguishable and the augmentations assignable to the appropriate folder. Hence the client application can relate input from any device to a specific folder and vice versa. To identify the interactive folders of the Smart Filing System, each folder is equipped with a set of unique IDs: in the cabinet, each folder is identified by the VoodooIO device ID and the front page of each folder by a unique Anoto paper. On the optional interactive table the ARTag Marker also provides a unique ID. The mapping of all mounted augmentations (and their IDs)

\footnotetext{
${ }^{4}$ http://office.microsoft.com/onenote
} 
to a real folder is stored by the client application. Finally, to establish a connection between the real folder and the associated virtual folder in OneNote the corresponding ID of an OneNote section is added to this set of IDs.

The interaction with the button and LED on the folder is facilitated by the VoodooIO service as described in the previous section. On manipulation of a device this service triggers an event that is handled by the client application. If a button is pressed the event handler queries for the OneNote section ID that corresponds to the device ID of the folder whose button was pressed. Accordingly it opens and displays the OneNote section that is linked to the real folder on which the pressed button is mounted.

The LED is used in two different ways. Either it indicates the currently viewed virtual folder in OneNote or it displays the search results of the enhanced search. In the first case, the client application listens for navigation events from OneNote, searches for the associated device ID and turns on the LED via the VoodooIO Service. In the second case, the LEDs are triggered as a result of the enhanced search. Because there was no way to modify the standard search feature of OneNote a custom search dialog was developed. Figure 9 depicts this dialog and the displayed search results on the monitor and in the filing cabinet. If a folder contains one or more search results, the corresponding LEDs will be turned on. A problem arises because OneNote provides a keyword search and hence the results aren't restricted to folders/sections only. Consequently, the indication of search results using one LED per folder can only show that something was found in this folder, and not the number of hits. To give the users a chance to distinguish between a folder with many hits and those with just a few, the rotary dial mounted on the cabinet is used. Internally, the folder information is sorted by the number of search results that have been found in each folder. By turning the rotary dial the number of highlighted folders is increased or decreased, thus folders with fewer hits can be hidden.

Another task of the software client is to synchronize the written notes on the front side of a folder with OneNote (see figure 10). A custom-developed Anoto pen driver, capable of handling multiple digital pens operating in streaming mode on a single computer, was used. The software client converts the stroke information into MS Digital $\mathrm{Ink}^{5}$ objects. These objects are finally imported to OneNote at an empty page in the section matching the selected interactive folder.

The storage and organization of digital documents and folders is handled by OneNote. As mentioned in the introduction, computer systems utilize metaphors of the physical office; OneNote organizes data in notebooks, section groups, sections, pages and sub-pages. Our interactive folders are linked with the sections of OneNote, because they best resemble physical folders. The OneNote API offers a rich set of functionalities for import and export of data. Since OneNote organizes its data in an XML format, it can be integrated easily into other applications.

\footnotetext{
${ }^{5} \mathrm{http} / / / \mathrm{msdn} . \mathrm{microsoft.com} / \mathrm{msdnmag} /$ issues/04/12/TabletPC/
}

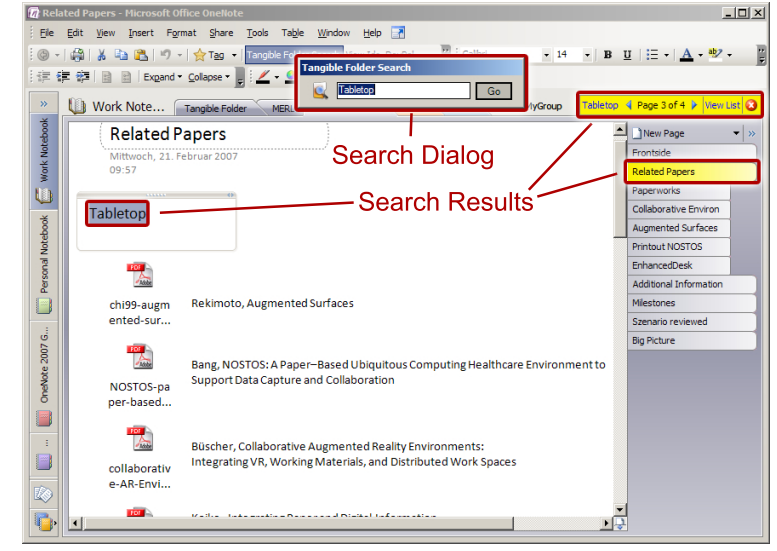

(a) Computer display

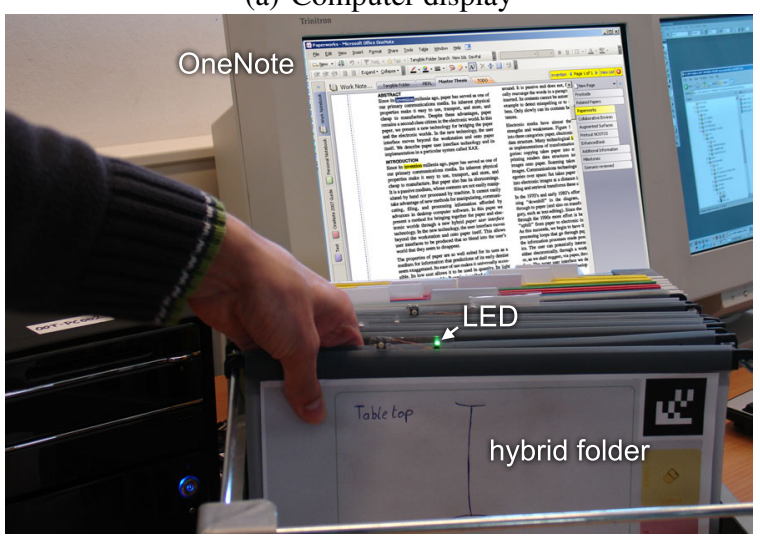

(b) Cabinet display

Figure 9. (a) An additional search dialog enables a simultanous search in the virtual and real document storage. (b) The lighting LED on the interactive folder indicates the actual selected folder in OneNote.

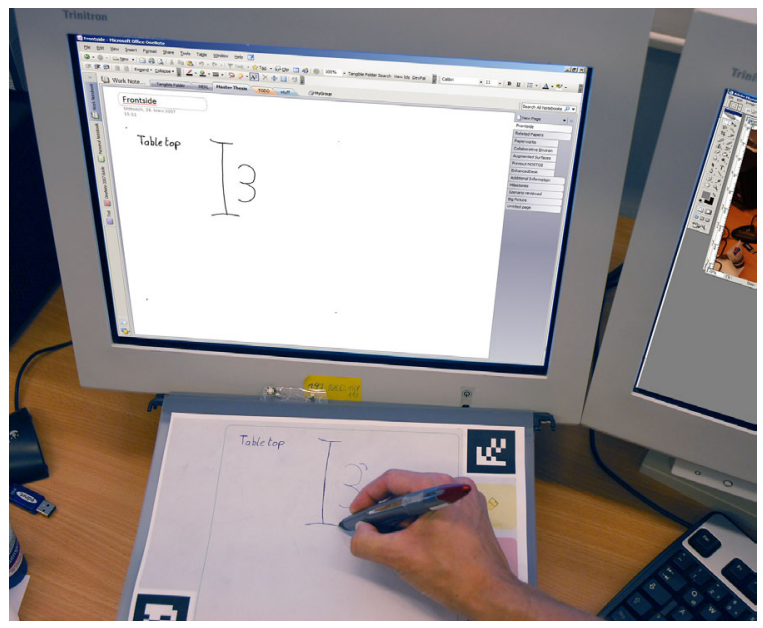

Figure 10. Annotations on the front side of an interactive folder are synchronized with the virtual DMS in real-time. 


\section{SCENARIOS}

The major function of our application is to make the process of storing, locating and retrieving physical documents more efficient, while also retaining the affordances of real printouts, which still have many advantages over digital documents [10]. In an office environment often a large number of printouts and physical documents are used, and many of them are filed in filing cabinets, the organization of which can potentially be complex.

\section{Searching}

An important aspect of filing documents is to be able to search and find them when they are needed. In a conventional filing system it is necessary to know in which order the folders are organized in the cabinet so that the required documents can be found quickly. A problem can however arise if a folder is misplaced. In this case the folder can only be found by manual browsing and searching through the cabinet, which can be a very time consuming task.

In the Smart Filing System these cases are handled by the following functionalities:

- The interactive folders register themselves the moment they are placed into a cabinet, establishing a connection to the computer system. Hence the system knows in which cabinet a particular folder is located. Therefore, it is not necessary to know the exact location of the folder, because each folder can be addressed through its ID, and a glowing LED can be quickly recognized by the human eye. Hence a folder is easy to find even when it is misplaced. In fact, it would be possible to place the folders in the cabinet randomly, with no order at all.

- By using the enhancement of the OneNote search feature, results are displayed directly at the cabinet as well as on the computer display. Folders containing search results are highlighted by their LEDs (cf. figure 9(b)). This keyword search allows folders containing the relevant documents to be found easily.

\section{Browsing}

Locating folders and documents through a query is not the only way to find them. Often it is just necessary to get the currently viewed folder. This is the case, for example, if someone wants to file a recently printed document in the currently viewed virtual folder, or if a printout of a viewed document is required.

As described earlier the Smart Filing System uses the LEDs to indicate the currently opened and displayed section in OneNote. The LEDs on the real folders are synchronized with the actual browsing through OneNote. Changing sections in OneNote causes the LED of the corresponding interactive folder to be turned on. Although the content of the virtual and physical folders are the same, in most cases the digital and real DMS can have a different order, and therefore it is necessary to know the exact mapping of the real and digital folders.

Additionally, our system supports a bi-directional link be- tween the interactive folders and the sections of OneNote. Users can either navigate through the different sections and trace the corresponding lighting on the interactive folders, or browse through the interactive folders and track the changes in OneNote. In this case, users simply have to press the button mounted near the LED of the interactive folder and OneNote opens and displays the corresponding section. This function can be very useful if the user finds a certain physical folder and is interested in other digital data, such as videos, audio files, etc. related to that folder.

\section{Annotation}

The front covers of physical folders are often used to write down some information about the content of the folder. This information is very useful for structuring the filing system. Documents that relate to others in some way are usually filed in the same folder. For example, in case of a property management company, all documents concerning a particular realty can be filed in the same folder. Hence the name and address of the realty might be written on the front side of the folder.

In our application, users can annotate the interactive folders on the front side too. Users can write on the annoation area of the folder by using the digital pens. The strokes are wirelessly sent to OneNote over Bluetooth and are stored in OneNote as ink strokes. Consequently, the information is accessible in both the real and digital world. Furthermore, the handwritten strokes are even searchable due to the real-time OCR of OneNote.

\section{EARLY USER FEEDBACK}

We conducted an initial pilot study with a few of our university colleagues, who were not affiliated with this project. The overall reaction from the participants was very positive, even though we used a prototype setup with one small cabinet. Users really liked the idea of searching documents using OneNote and browsing through the folders. Although the participants had never used OneNote before, they found the information they were searching for quickly. It was easy for them to find folders by using either the enhanced search feature, or the synchronized navigation. The participants said that the glowing LED is a great support because it immediately shows the folders that they are looking for. In our testing environment, we only used 5 folders. In a real-world setup, people often have to find a folder in a filing cabinet archiving hundreds of folders (e.g. see figure 1), which would demonstrate the benefits of our system more clearly.

Participants also liked the idea of making notes on the front page of each folder, which automatically was transferred to a corresponding OneNote folder. One of the participants also had the idea of using the Anoto pattern on every document inside the folder, which would allow more interactivity with the real and digital documents. All paper annotations would then be automatically transferred as digital strokes into OneNote. Another feature, which was well received by all the participants, was the link between the real folder and the digital world. Users often need to find a certain document/folder and want to see the corresponding digital document. In our 
setup, participants only have to click the corresponding folders button which automatically triggers the OneNote folder containing the additional information to be opened.

When filing documents into folders it was also easy to locate the correct folder. Most participants used the synchronized navigation feature to light the LED on the linked physical folder. Some users also had concerns about misplaced documents because they might be difficult to find them again.

Finally, in some (rare) cases, we had problems with the connection contacts of the physical folder with the rail of the filing cabinet - which caused problems while searching for content. This effect mainly occurred once the folder was too light, and hence the current linkage between to the cabinet broke.

\section{CONCLUSIONS}

In this paper we presented the Smart Filing System, which provides a combination of digital and physical interfaces to an augmented filing cabinet system. Our approach links the virtual and physical document storage and retrieval capabilities of the traditional filing cabinets and folders to that of a computer system. Our system hooks into Microsoft OneNote for interfacing the virtual and physical filing cabinets, folders, pages, and content at the software level, while the hardware link between the augmented filing cabinet and the computer system is achieved using VoodooIO. Additional links between the physical and digital documents are created using the Anoto pens and paper.

We are currently optimizing our hardware and software systems to allow a large number of folders to be added to our system, so that more complex scenarios of use can be investigated. A further enhancement would be to add RFID technology to our folders(similar to [6]) to track documents inside the folder. This would ease the problem of misplaced documents. We are also planning to incorporate other tangible interfaces which can be connected with 3rd party applications to our existing filing cabinet environment, and by doing so further improve computer supported work, particularly in collaborative tabletop settings.

\section{ACKNOWLEDGEMENTS}

The augmented filing cabinet and interactive folders system was developed by Matthew Jervis using the VoodooIO interface technology from Lancaster University. The Smart Filing System client software was developed by Thomas Seifried using the Microsoft OneNote. We would also like to thank Chen Shia from MERL for donating the DiamondTouch table, the people from ANOTO and Maxell for the great support.

\section{REFERENCES}

1. Majed AbuSafiya and Subhasish Mazumdar. Accommodating paper in document databases. In Proc. of the 2004 ACM sym. on Doc. engi., pages 155-162, Milwaukee, Wisconsin, USA, 2004. ACM Press.

2. Damian Arregui, Christer Fernstrom, Francois Pacull, Gilbert Rondeau, Jutta Willamowski, Elisabeth
Crochon, and Francois Favre-Reguillon. Paper-based communicating objects in the future office. In Proc. Smart Object conf., Grenoble, France, 2003.

3. Mark Fiala. Artag, a fiducial marker system using digital techniques. In CVPR '05: Proc. of the 2005 IEEE Comp. Society Conf. on Comp. Vision and Pattern Recognition (CVPR'05) - Volume 2, pages 590-596, Washington, DC, USA, 2005. IEEE Computer Society.

4. Andrew Glassner. Everyday computer graphics. IEEE Comp. Graph. Appl., 23(6):76-82, 2003.

5. Saul Greenberg and Michael Boyle. Customizable physical interfaces for interacting with conventional applications. In UIST '02: Proc. of the 15th annual ACM sym. on User interface software and technology, pages 31-40, New York, NY, USA, 2002. ACM Press.

6. François Guimbretière. Paper augmented digital documents. In UIST '03: Proc. of the 16th annual ACM sym. on User interface software and technology, pages 51-60, New York, NY, USA, 2003. ACM Press.

7. Hiroshi Ishii and Brygg Ullmer. Tangible bits: towards seamless interfaces between people, bits and atoms. In CHI '97: Proc. of the SIGCHI conf. on Human factors in comp. sys., pages 234-241, New York, NY, USA, 1997. ACM Press.

8. Walter Johnson, Herbert Jellinek, Jr. Leigh Klotz, Ramana Rao, and Stuart Card. Bridging the paper and electronic worlds: the paper user interface. In INTERCHI '93: Proc. of the INTERCHI'93 conf. on Human factors in comp. sys., pages 507-512, Amsterdam, The Netherlands, 1993. IOS Press.

9. Hidkei Koike, Yoichi Sato, and Yoshinori Kobayashi. Integrating paper and digital information on enhanceddesk: a method for realtime finger tracking on an augmented desk system. ACM Trans. Comp.-Human Interaction, 8(4):307-322, 2001.

10. Chunyuan Liao, François Guimbretière, and Ken Hinckley. Papiercraft: a command system for interactive paper. In UIST '05: Proc. of the 18th annual ACM sym. on User interface software and technology, pages 241-244, New York, NY, USA, 2005. ACM Press.

11. United States National Museum. Report on the Progress and Condition of the U.S. Nation Museum for the Year Ending June 30. G.P.O., 1939.

12. Abigail J. Sellen and Richard H.R. Harper. The Myth of the Paperless Office. MIT Press, Cambridge, MA, USA, 2003.

13. Nicolas Villar and Hans Gellersen. A malleable control structure for softwired user interfaces. In TEI '07:

Proc. of the 1st int. conf. on Tangible and emb. inter., pages 49-56, New York, NY, USA, 2007. ACM Press.

14. Pierre Wellner. Interacting with paper on the digitaldesk. Commun. ACM, 36(7):87-96, 1993. 